\title{
Quantitative analysis of a Māori and Pacific admission process on first-year health study
}

\author{
Elana Curtis ${ }^{1 *}$, Erena Wikaire', Yannan Jiang ${ }^{2}$, Louise McMillan², Robert Loto ${ }^{1}$, Airini ${ }^{3}$ and Papaarangi Reid ${ }^{1}$
}

\begin{abstract}
Background: Universities should provide flexible and inclusive selection and admission policies to increase equity in access and outcomes for indigenous and ethnic minority students. This study investigates an equity-targeted admissions process, involving a Multiple Mini Interview and objective testing, advising Māori and Pacific students on their best starting point for academic success towards a career in medicine, nursing, health sciences and pharmacy.

Methods: All Māori and Pacific Admission Scheme (MAPAS) interviewees enrolled in bridging/foundation or degree-level programmes at the University of Auckland were identified (2009 to 2012). Generalised linear regression models estimated the predicted effects of admission variables (e.g. MAPAS Maths Test; National Certificate in Educational Achievement (NCEA) Rank Score; Any 2 Sciences; Followed MAPAS Advice) on first year academic outcomes (i.e. Grade Point Average (GPA) and Passes All Courses) adjusting for MAPAS interview year, gender, ancestry and school decile.

Results: 368 First Year Tertiary (bridging/foundation or degree-level) and 242 First Year Bachelor (degree-level only) students were investigated. NCEA Rank Score (estimate 0.26, Cl: 0.18-0.34, $p<0.0001$ ); MAPAS Advice Followed (1.26, Cl: 0.18-1.34, $p=0.0002)$; Exposure to Any 2 Sciences (0.651, Cl: 0.15-1.15, $p=0.012)$; and MAPAS Mathematics Test $(0.14, \mathrm{Cl}: 0.02-0.26, p=0.0186)$ variables were strongly associated with an increase in First Year Tertiary GPA. The odds of passing all courses in First Year Tertiary study was 5.4 times higher for students who Followed MAPAS Advice (Cl: 2.35-12.39; $p<0.0001)$ and 2.3 times higher with Exposure to Any Two Sciences (Cl: 1.15-4.60; $p=0.0186)$. First Year Bachelor students who Followed MAPAS Advice had an average GPA that was 1.1 points higher for all eight (Cl: $0.45-1.73 ; p=0.0009)$ and Core 4 courses (Cl: $0.60-2.04 ; p=0.0004)$.

Conclusions: The MAPAS admissions process was strongly associated with positive academic outcomes in the first year of tertiary study. Universities should invest in a comprehensive admissions process that includes alternative entry pathways for indigenous and ethnic minority applicants.

Keywords: Admission, Selection, Indigenous, Ethnic minority, Health professional, Higher education, Widening participation, Workforce development, Māori, Pacific
\end{abstract}

\section{Background}

Worldwide, tertiary institutions are attempting to widen participation to historically underserved populations including indigenous and ethnic minority students [1] Often driven by social inclusion and social accountability policies, universities have devised a number of strategies to increase diversity. Within an indigenous and ethnic minority health workforce context, a pipeline approach

\footnotetext{
*Correspondence: e.curtis@auckland.ac.nz

${ }^{1}$ Te Kupenga Hauora Mãori, Faculty of Medical and Health Sciences, University of Auckland, Private Bag 92015, Auckland, New Zealand Full list of author information is available at the end of the article
}

is recommended to address well-known barriers to accessing and succeeding in university-level studies. A pipeline approach often includes early exposure interventions aimed at raising aspirations and academic preparation for a career in health [2-4]; addressing educational disadvantage via the provision of bridging/ foundation programmes $[5,6]$ and improving student performance by providing comprehensive support programmes [7-9]. Given the highly competitive context of health professional programme selection, it is also recommended that universities provide more flexible and 
Table 1 Definition of terms used within the FMHS context

\begin{tabular}{ll}
\hline Term & Definition \\
\hline $\begin{array}{l}\text { Certificate in Health } \\
\text { Sciences }\end{array}$ & $\begin{array}{l}\text { A 1-year bridging foundation level programme for Māori and Pacific students that provides an alternative entry pathway to } \\
\text { the first year of bachelor degree level undergraduate FMHS health programmes }\end{array}$ \\
First Year Tertiary & $\begin{array}{l}\text { The first year in which a student enrols in a form of study provided by the tertiary institution (e.g. Certificate in Health } \\
\text { Sciences or First Year Bachelor) } \\
\text { First Year Bachelor }\end{array}$ \\
\hline
\end{tabular}

inclusive selection and admission policies for students from underserved populations $[1,10]$.

Universities have a choice of selection tools that can be used to inform student admission including prior academic performance, interview scores and results from aptitude tests. Both cognitive and non-cognitive tools are used by universities when selecting students; however it is arguable that prior academic performance remains a dominant tool for medical selection in many universities [11]. Given this reality, indigenous and ethnic minority students are required to aim to achieve a high level of academic performance within the pathways used for future selection into medical or health professional programmes of study [12]. Unfortunately, students from underserved populations are less likely to receive access to science-rich subjects and are more likely to leave high school with lower qualifications than their peers $[5,10,13]$. Providing an admissions process that can determine whether indigenous and ethnic minority applicants are academically (and socially) ready to achieve success in pre-medical degree pathways and the provision of alternative entry pathways is recommended for tertiary institutions committed to widening participation $[14,15]$.

An extensive body of research identifies the tertiary conditions and factors that impact on academic success within the first year of study at university [16-20]. Indicators of prior academic performance such as: secondary school grade point averages [21]; secondary school factors including markers of socio-geographic status (e.g. school decile) [22]; and student characteristics (e.g. autonomy, confidence, motivation, control) $[17,23]$ have been identified as important factors impacting on academic performance in the first year of study. In addition, factors associated with the environment of the tertiary institution also impact on student engagement; such factors include: opportunities for teachers and students to engage with each other [18]; levels of institutional support to provide environments conducive to learning [20]; and the provision of academic, social and personal support [16].

To date, few studies have explored the effect of equitytargeted admission processes on the academic performance of indigenous and ethnic minority students in their first year of tertiary study. As a result, tertiary institutions have little empirical evidence to understand the effect of equity-targeted selection processes and whether such initiatives are likely to support a widening participation agenda.

This article explores the predictive effect of admission variables associated with an equity-targeted admission process on academic outcomes for Māori (the indigenous peoples of Aotearoa New Zealand) and Pacific (a heterogeneous composite of peoples with Pacific nation ancestry born and/or living in New Zealand) applicants applying under the Māori and Pacific Admission Scheme (MAPAS) to the Faculty of Medical and Health Sciences (FMHS) at the University of Auckland (UoA).

\section{Methods}

\section{FMHS entry pathways}

Admission into FMHS health professional programmes is generally via direct entry into First Year Bachelor level undergraduate study for those applicants who meet the necessary entry requirements [24]. The FMHS also offers a one-year, MAPAS-specific bridging/foundation programme, the Certificate in Health Sciences (CertHSc) through which Mãori and Pacific students who achieve a CertHSc GPA above B+ can gain alternative entry into First Year Bachelor undergraduate study. Hence, Māori and Pacific First Year Tertiary students within FMHS could either be enrolled in the CertHSc bridging foundation programme, or, the first year of bachelor level study (Table 1). The first year of bachelor level study also acts as a 'pre-medical' year prior to admission into the FMHS Medical programme in year 2. Table 1 provides definitions of the Certificate in Health Sciences, First Year Tertiary, and First Year Bachelor terms used within this study (Table 1).

\section{Māori and Pacific Admission Scheme (MAPAS)}

MAPAS operates an equity-targeted admissions process for applicants with indigenous Māori and Pacific ancestry. The process aims to gather a broad range of information about Māori and Pacific applicant preparation for tertiary health study. The December interview process involves a Multiple Mini Interview (MMI), an English test and a mathematics test.

The MMI is an alternative form of admission interview that aims to reduce interviewer bias by consisting of a number of short interview stations with multiple interviewers. The MMI has been shown to be reliable, 
Table 2 Descriptive summary of first year tertiary and first year bachelor student demographic and outcome variables

\begin{tabular}{|c|c|c|c|c|}
\hline \multirow{3}{*}{$\begin{array}{l}\text { Descriptive summary } \\
\text { variables } \\
\text { Continuous variables }\end{array}$} & \multicolumn{2}{|c|}{ First year tertiary students } & \multicolumn{2}{|c|}{ First year bachelor students } \\
\hline & \multicolumn{2}{|c|}{$2009-2012(n=368)$} & \multicolumn{2}{|c|}{$2009-2012(n=242)$} \\
\hline & Mean & $\pm S D$ & Mean & $\pm S D$ \\
\hline Age (Years $\pm S D)$ & 19.2 & 4.2 & 19.0 & 3.9 \\
\hline Categorical variables & $n$ & $\%$ & $n$ & $\%$ \\
\hline \multicolumn{5}{|l|}{ Year of admission } \\
\hline 2009 & 70 & 19 & 26 & 11 \\
\hline 2010 & 95 & 26 & 69 & 29 \\
\hline 2011 & 108 & 29 & 79 & 32 \\
\hline 2012 & 95 & 26 & 68 & 28 \\
\hline \multicolumn{5}{|l|}{ Gender } \\
\hline Female & 248 & 67 & 160 & 66 \\
\hline Male & 120 & 33 & 82 & 34 \\
\hline \multicolumn{5}{|l|}{ Ancestry } \\
\hline Māori & 137 & 37 & 89 & 37 \\
\hline Pacific & 210 & 57 & 138 & 57 \\
\hline Both Māori and Pacific & 21 & 6 & 15 & 6 \\
\hline \multicolumn{5}{|l|}{ School Decile } \\
\hline High (8-10) & 82 & 24 & 59 & 26 \\
\hline Medium (4-7) & 144 & 41 & 98 & 43 \\
\hline Low (1-3) & 123 & 35 & 71 & 31 \\
\hline Missing & 19 & - & 14 & - \\
\hline Continuous variables & Mean & $\pm S D$ & Mean & $\pm S D$ \\
\hline \multicolumn{5}{|l|}{ Grade Point Average (GPA) } \\
\hline Eight Courses & 4.3 & 2.0 & 4.1 & 2.1 \\
\hline Core 4 Courses & - & - & 3.8 & 2.4 \\
\hline Categorical variables & $n$ & $\%$ & $n$ & $\%$ \\
\hline \multicolumn{5}{|l|}{ Passes All Eight Courses } \\
\hline Yes & 276 & 75 & 145 & 60 \\
\hline No & 92 & 25 & 97 & 40 \\
\hline \multicolumn{5}{|l|}{ Passes All Core 4 Courses } \\
\hline Yes & - & - & 154 & 64 \\
\hline No & - & - & 88 & 36 \\
\hline
\end{tabular}

acceptable and feasible in a variety of tertiary health study contexts [25]. In building on the original pilot of the MMI [26], other studies have taken advantage of the intended benefit of the flexibility of station development in their own contexts $[27,28]$. Whilst the original authors aimed to assess suitability of applicants as health professionals, the MAPAS MMI aims to assess Māori and Pacific applicant preparation for and potential to succeed in FMHS programmes. In the MAPAS context, the MMI has been redeveloped to include four 8-min stations assessing career aspirations; academic preparation; family support and student information. The MAPAS mathematics and English testing are used in addition to the MAPAS MMI to objectively assess academic numeracy and literacy skills. Using MMI and testing information, two assessments are made about: 1) potential to succeed within the CertHSc, and 2) potential to succeed within the Bachelor of: Health Sciences; Science (Biomedicine) ${ }^{1}$; Nursing; or Pharmacy. Potential to succeed is assessed as: pass, borderline or fail (objective testing) for the English and mathematics testing and few, some, or major concerns (subjective testing) for each MMI station. A MAPAS Recommendations Team reviews the combination of results and provides a provisional MAPAS recommendation (advice regarding the applicant's recommended best starting point given 
Table 3 Descriptive summary of first year tertiary and first year bachelor student predictor variables

\begin{tabular}{|c|c|c|c|c|}
\hline \multirow{3}{*}{$\begin{array}{l}\text { Predictors } \\
\text { Continuous variables }\end{array}$} & \multicolumn{2}{|c|}{ First year tertiary students } & \multicolumn{2}{|c|}{ First year bachelor students } \\
\hline & \multicolumn{2}{|c|}{$2009-2012(n=368)$} & \multicolumn{2}{|c|}{$2009-2012(n=242)$} \\
\hline & $n$ & Mean $\pm S D$ & $n$ & Mean $\pm S D$ \\
\hline \multicolumn{5}{|l|}{ MAPAS testing } \\
\hline Mathematics test & 241 & $79.0 \pm 18.3$ & 241 & $80.4 \pm 18.3$ \\
\hline English test & 241 & $68.4 \pm 13.6$ & 241 & $70.6 \pm 12.8$ \\
\hline Categorical variables & $n$ & $\%$ & $n$ & $\%$ \\
\hline \multicolumn{5}{|l|}{ CertHSC MMI } \\
\hline \multicolumn{5}{|l|}{ Whānau Support } \\
\hline$F C^{d}$ & 305 & 83 & 208 & 86 \\
\hline SMC & 63 & 17 & 34 & 14 \\
\hline \multicolumn{5}{|c|}{ Academic Preparation } \\
\hline $\mathrm{FC}$ & 306 & 83 & 210 & 87 \\
\hline SMC & 62 & 17 & 32 & 13 \\
\hline \multicolumn{5}{|l|}{ Career Aspirations } \\
\hline $\mathrm{FC}$ & 296 & 80 & 202 & 84 \\
\hline SMC & 72 & 20 & 40 & 16 \\
\hline \multicolumn{5}{|l|}{ Student Information } \\
\hline $\mathrm{FC}$ & 295 & 80 & 206 & 85 \\
\hline SMC & 73 & 20 & 36 & 15 \\
\hline \multicolumn{5}{|l|}{ Bachelor MMI } \\
\hline \multicolumn{5}{|l|}{ Whānau Support } \\
\hline FC & 250 & 68 & 178 & 74 \\
\hline SMC & 118 & 32 & 64 & 26 \\
\hline \multicolumn{5}{|c|}{ Academic Preparation } \\
\hline FC & 207 & 56 & 157 & 65 \\
\hline SMC & 161 & 44 & 85 & 35 \\
\hline \multicolumn{5}{|l|}{ Career Aspirations } \\
\hline FC & 296 & 80 & 125 & 52 \\
\hline SMC & 72 & 20 & 117 & 48 \\
\hline \multicolumn{5}{|c|}{ Student Information (missing $=1$ ) } \\
\hline FC & 205 & 56 & 146 & 61 \\
\hline SMC & 162 & 44 & 95 & 39 \\
\hline \multicolumn{5}{|c|}{ December Recommendation (Provisional) } \\
\hline CertHSC & 197 & 55 & 112 & 48 \\
\hline Bachelor & 131 & 37 & 109 & 47 \\
\hline Not FMHS & 28 & 8 & 12 & 5 \\
\hline Missing & 12 & - & 9 & - \\
\hline Continuous variables & $n$ & Mean $\pm S D$ & $n$ & Mean $\pm S D$ \\
\hline \multicolumn{5}{|l|}{ School Results (NCEA) ${ }^{a}$} \\
\hline Rank Score & 291 & $190.5 \pm 51.3$ & 194 & $201.8 \pm 52.7$ \\
\hline L3 English ${ }^{b}$ & 225 & $16.7 \pm 5.8$ & 150 & $17.7 \pm 5.6$ \\
\hline L3 Biology & 260 & $15.4 \pm 6.1$ & 172 & $16.8 \pm 5.9$ \\
\hline L3 Chemistry & 233 & $14.6 \pm 7.1$ & 165 & $15.7 \pm 7.0$ \\
\hline L3 Physics & 132 & $15.3 \pm 7.8$ & 99 & $16.6 \pm 7.8$ \\
\hline
\end{tabular}


Table 3 Descriptive summary of first year tertiary and first year bachelor student predictor variables (Continued)

\begin{tabular}{|c|c|c|c|c|}
\hline L3 Maths & 266 & $24.2 \pm 13.7$ & 177 & $26.3 \pm 14.5$ \\
\hline \multicolumn{5}{|l|}{ Categorical variables } \\
\hline Any 2 sciences $(\mathrm{NCEA}, \mathrm{CIE}, \mathrm{IB})^{\mathrm{c}}$ & $n$ & $\%$ & $n$ & $\%$ \\
\hline Yes & 244 & 66 & 171 & 85 \\
\hline No & 55 & 15 & 31 & 15 \\
\hline AA/no school results & 69 & - & 40 & - \\
\hline \multicolumn{5}{|l|}{ Followed advice } \\
\hline Yes & 315 & 88.0 & 196 & 83 \\
\hline No & 43 & 12.0 & 39 & 17 \\
\hline Missing & 10 & - & 7 & \\
\hline \multicolumn{5}{|l|}{ January Recommendation (Final) } \\
\hline CertHSc & 256 & 71.5 & 137 & 58 \\
\hline Bachelor & 95 & 26 & 91 & 39 \\
\hline Not FMHS & 7 & 2 & 7 & 3 \\
\hline Missing & 10 & - & 7 & - \\
\hline
\end{tabular}

Rank Score and L3 subject results analysis was completed for applicants who completed the National Certificate in Educational Achievement (NCEA) only. Excludes Cambridge International Exam (CIE), International Baccalaureate (IB), International students, alternative admission applicants and missing data b 23 subject missing data includes those NCEA applicants who did not enrol in that particular subject

${ }^{C}$ Any 2 sciences was calculated for all applicants who had available subject results for any two of the three applied science subjects (Physics, Biology, and Chemistry). $\mathrm{N}$ for any 2 sciences differs from Rank Score as it does not exclude CIE, IB, International, or alternative admission students

${ }^{\mathrm{d}}$ FC Few concerns, SMC Some or major concerns

their intended health career) for applicants (and families) on the day of their interview. Recommended starting points are reflected within three categories: (1) Bachelor i.e. start at degree-level; (2) CertHSc i.e. start at bridging/ foundation; or (3) Not FMHS i.e. start in a pathway not provided by FMHS (likely to need further academic preparation not offered by the FMHS). Following the release of secondary school results in January, all information is re-reviewed and a final MAPAS recommendation is provided. MAPAS recommendations are not binding if an applicant has met guaranteed entry criteria for any FMHS programme. In this context, the applicant can choose to follow MAPAS advice (or not) ${ }^{2}$.

\section{Methodology}

This study used a Kaupapa Mãori Research (KMR) approach, broadly defined and responsive to Pacific research methodologies $[29,30]$. This approach recognises that issues associated with power, privilege and agency within society are hypothesised to act similarly on both Māori and Pacific students [31, 32]. In this instance KMR aims to: ensure research outputs are positive for Māori and Pacific students; explicitly challenge 'victim blame' or 'cultural deficit' analyses that may blame Māori or Pacific students for educational failure; and provide a structural analysis to promote institutional change targeting Māori and Pacific student success [14, 33]. This research was led by senior Mãori and Pacific researchers with input from a FMHS advisory group.

\section{Study design}

The predictive effect of MAPAS admission process variables on academic outcomes in the first year of tertiary study was explored. Applicant data were obtained from the MAPAS admissions database and the university's centralised student data management system for all MAPAS interviewees (2008 - 2011) who subsequently enrolled in relevant tertiary health programmes $(2009$ - 2012) within the FMHS at the UoA. Approval to complete this research was granted by the University of Auckland Human Participant Ethics Committee (Ref 8110 ). As per ethics protocols, written informed consent was not required for this research project due to the use of secondary administrative data sources. All secondary data obtained from these datasets were de-identified by an independent research member with no student contact or teaching responsibilities and data analysis occurred via a coding system. Two student cohorts are identified: First Year Tertiary Students i.e. students enrolled in either the CertHSc or the first year of a bachelor programme in the year following their MAPAS interview; and First Year Bachelor Students i.e. students enrolled in a bachelor programme in either the first or second year following their MAPAS interview (may include CertHSc graduates).

\section{Variables}

Demographic variables include: Year of Admission (2009-2012); Gender (Female, Male); Ancestry (Māori, Pacific, Both) and School Decile (High, Medium and Low). Secondary schools with a mid-low decile rating 
Table 4 Univariate regression analysis results - GPA eight courses

\begin{tabular}{|c|c|c|c|c|}
\hline \multirow[t]{3}{*}{ Predictors } & \multicolumn{2}{|l|}{ First year tertiary students } & \multicolumn{2}{|l|}{ First year bachelor students } \\
\hline & \multicolumn{2}{|l|}{$2009-2012(n=368)$} & \multicolumn{2}{|l|}{$2009-2012(n=242)$} \\
\hline & Mean estimate $(95 \% \mathrm{Cl})$ & $P$ value & Mean estimate $(95 \%$ Cl) & $P$ value \\
\hline \multicolumn{5}{|l|}{ GPA Eight Courses } \\
\hline \multicolumn{5}{|c|}{ Any 2 sciences $(\mathrm{NCEA}, \mathrm{CIE}, \mathrm{IB})^{* *}$} \\
\hline No & 0.00 & & 0.00 & \\
\hline Yes & $0.971(0.44,1.50)$ & $0.0004^{*}$ & $0.912(0.17,1.65)$ & 0.0169 \\
\hline \multicolumn{5}{|l|}{ Followed advice } \\
\hline No & 0.00 & & 0.00 & \\
\hline Yes & $0.78(0.18,1.38)$ & $0.0109^{*}$ & $0.84(0.17,1.51)$ & $0.0147^{*}$ \\
\hline \multicolumn{5}{|l|}{ CertHSC MMI } \\
\hline \multicolumn{5}{|l|}{ Whānau Support } \\
\hline $\mathrm{FC}^{\mathrm{a}}$ & 0.00 & & 0.00 & \\
\hline SC & $0.14(-0.43,0.71)$ & 0.6201 & $0.66(-0.12,1.44)$ & 0.0972 \\
\hline MC & $-1.5(-2.98,-0.02)$ & 0.0475 & $-1.41(-4.24,1.42)$ & 0.3290 \\
\hline \multicolumn{5}{|c|}{ Academic Preparation } \\
\hline FC & 0.00 & & 0.00 & \\
\hline SC & $-0.27(-0.87,0.33)$ & 0.3799 & $-0.29(-1.18,0.60)$ & 0.5254 \\
\hline MC & $0.56(-0.50,1.62)$ & 0.2989 & $0.93(-0.66,2.52)$ & 0.2531 \\
\hline \multicolumn{5}{|l|}{ Career Aspirations } \\
\hline FC & 0.00 & & 0.00 & \\
\hline SC & $-0.83(-1.39,-0.28)$ & $0.0035^{*}$ & $-1.10(-1.90,-0.29)$ & $0.0081^{*}$ \\
\hline$M C$ & $-0.28(-1.56,1.00)$ & 0.6676 & $1.12(-0.53,2.77)$ & 0.1833 \\
\hline \multicolumn{5}{|c|}{ Student Information } \\
\hline FC & 0.00 & & 0.00 & \\
\hline SC & $1.28(0.72,1.84)$ & 0.3100 & $-0.47(-1.29,0.34)$ & 0.2572 \\
\hline$M C$ & $-0.29(-1.60,1.02)$ & 0.0559 & $2.06(-0.26,4.37)$ & 0.0834 \\
\hline \multicolumn{5}{|l|}{ Bachelor MMI } \\
\hline Whānau Support & & & 0.00 & \\
\hline FC & 0.00 & & $0.03(-0.65,0.71)$ & \\
\hline SC & $-0.07(-0.56,0.42)$ & 0.2503 & $0.59(-0.56,1.74)$ & 0.346 \\
\hline$M C$ & $-0.38(-1.22,0.46)$ & 0.4301 & & 0.586 \\
\hline \multicolumn{5}{|c|}{ Academic Preparation } \\
\hline FC & 0.00 & & 0.00 & \\
\hline SC & $-0.08(-0.59,0.43)$ & 0.2601 & $-0.04(-0.71,0.64)$ & 0.345 \\
\hline$M C$ & $-0.15(-0.76,0.46)$ & 0.3112 & $0.05(-0.85,0.96)$ & 0.463 \\
\hline \multicolumn{5}{|l|}{ Career Aspirations } \\
\hline FC & 0.00 & & 0.00 & \\
\hline SC & $-0.73(-1.18,-0.28)$ & 0.2315 & $-0.77(-1.37,-0.17)$ & 0.307 \\
\hline$M C$ & $-0.79(-1.40,-0.19)$ & 0.3076 & $-0.74(-1.56,0.08)$ & 0.419 \\
\hline \multicolumn{5}{|c|}{ Student Information } \\
\hline FC & 0.00 & & 0.00 & \\
\hline SC & $-0.04(-0.50,0.41)$ & 0.2344 & $-0.13(-0.73,0.47)$ & 0.306 \\
\hline$M C$ & $-0.25(-0.95,0.45)$ & 0.3564 & $-1.23(-2.20,-0.25)$ & 0.497 \\
\hline
\end{tabular}


Table 4 Univariate regression analysis results - GPA eight courses (Continued)

\begin{tabular}{|c|c|c|c|c|}
\hline \multicolumn{5}{|l|}{ Continuous variables } \\
\hline \multicolumn{5}{|l|}{ School Results (NCEA)* } \\
\hline Rank Score (per 20 pt increase) & $0.26(0.18,0.34)$ & $<0.0001^{*}$ & $0.36(0.26,0.44)$ & $<0.0001^{*}$ \\
\hline L3 English^ & $-0.005(-0.09,0.08)$ & 0.912 & $-0.006(-0.09,0.08)$ & 0.9014 \\
\hline L3 Biology & $0.051(-0.03,0.14)$ & 0.249 & $0.034(-0.06,0.13)$ & 0.4711 \\
\hline L3 Chemistry & $0.001(-0.08,0.08)$ & 0.987 & $-0.044(-0.13,0.04)$ & 0.3039 \\
\hline L3 Physics & $0.091(0.03,0.15)$ & $0.004^{*}$ & $0.06(-0.004,0.13)$ & 0.0708 \\
\hline L3 Maths & $0.008(-0.03,0.05)$ & 0.664 & $0.036(-0.01,0.08)$ & 0.0964 \\
\hline MAPAS Maths test (per $10 \%$ increase) & $0.23(0.11,0.35)$ & $0.0002^{*}$ & $0.18(0.03,0.34)$ & $0.0233^{*}$ \\
\hline MAPAS English test(per $10 \%$ increase) & $0.09(-0.09,0.26)$ & 0.324 & $0.05(-0.19,0.29)$ & 0.6834 \\
\hline
\end{tabular}

$\wedge$ L3 subject missing data includes those NCEA applicants who did not enrol in that particular subject

*Adjusted for MAPAS interview year, gender, ancestry and school decile. For GPA (a continuous outcome variable), its mean change associated with the change in alinear predictor was estimated with $95 \%$ confidence interval. For a continuous predictor variable, this gave the difference in means with either 20 point (NCEA Rank Score) or $10 \%$ (MAPAS Maths percentage mark) increase in the predictor. For a categorical predictor, this gave the difference in means between the current and reference categories (i.e. yes vs. no). The null hypothesis was that there was no change in the mean response (i.e. $\Delta=0$ )

${ }^{*} \mathrm{NCEA}=$ National Certificate in Educational Achievement, CIE = Cambridge International Exam, IB = International Baccalaureate

${ }^{a}$ FC Few concerns, SMC Some or major concerns

have been linked to higher levels of deprivation associated with reduced access to, and outcomes from, tertiary education [34] (Table 2).

Admission predictor variables include: MAPAS Testing results (\%); MMI Station results (Some or Major Concerns (SMC) versus Few Concerns (FC)); Provisional December Recommendation (CertHSc, Bachelor, Not FMHS); secondary school results including New Zealand's NCEA Rank Score ${ }^{3}$ (out of 320); Level 3 NCEA Subject Credits (number of credits achieved in English, biology, chemistry, physics, mathematics); Exposure to Any 2 Sciences of senior biology, chemistry or physics (yes, no) ${ }^{4}$; Followed MAPAS Advice (yes, no); and Final January Recommendation made in January (CertHSc, Bachelor, Not FMHS).

Academic outcome variables include: Grade Point Average (GPA) Eight Courses, 0-9 (i.e. GPA achieved across a total of eight courses over the year); GPA Core 4 Courses, 0-9 (i.e. GPA achieved across four core courses $^{5}$ taken in the first year of bachelor study that are specifically assessed for selection into second year medicine at the UoA); Passes All Courses, yes/no (i.e. across total of eight courses); Passes All Core 4 Courses, yes/no (i.e. across the four core courses).

\section{Statistical analysis}

All downloaded data were recorded in Microsoft Office Excel spread sheets. Statistical analyses were performed using SAS version 9.3 (SAS Institute, Cary, NC, USA). Continuous variables were presented as mean and standard deviation (SD); categorical variables as frequencies (n) and percentages (\%) (Tables 2 and 3). Generalised linear and logistic regression models were used to estimate the predicted effects of individual admission variables on academic outcomes (i.e. GPA and Passes All); adjusting for pre-defined demographic variables (i.e. MAPAS interview year, gender, ancestry and school decile) (Tables 4, 5, 6 and 7). Admission variables that showed significant single predictive effect (i.e. MAPAS Maths Test, NCEA Rank Score, Any 2 Sciences and Followed MAPAS Advice) were included in the multiple regression analyses to determine their joint effects on the academic outcomes of interest (Tables 8 and 9). All statistical tests were two-sided at $5 \%$ significance level.

\section{Results}

\section{Descriptive variables}

A total of 368 students were identified in the First Year Tertiary cohort. Of these, 37 \% were Māori, 57 \% Pacific and $6 \%$ had Both Māori and Pacific ancestry. Two thirds were female $(67 \%)$, the mean age was 19.2 years (SD $4.2 \%$ ) and $70 \%$ or more came from a secondary school with a medium or low school decile (representing more deprived communities). The First Year Bachelor cohort had a total of 242 students with a similar demographic profile to First Year Tertiary students (Table 2).

\section{Predictor variables}

\section{Mathematics and english testing}

The First Year Tertiary cohort had a mean percentage mark for the mathematics test of $79.0 \%$ (SD $18.3 \%$ ) and $68.4 \%$ (SD $13.6 \%$ ) for the English test. This represents a borderline-fail result for bachelor-level study and a pass result for CertHSc-level study as the best starting point of entry across both assessments. The First Year Bachelor cohort had a slightly higher mean mark for both the mathematics (80.4\%, SD $18.3 \%$ ) and English tests $(70.6 \%$, SD $12.8 \%$ ) (Table 3 ). 
Table 5 Univariate regression analysis results - GPA core 4 courses

\begin{tabular}{|c|c|c|c|c|}
\hline \multirow[t]{3}{*}{ Predictors } & \multirow{2}{*}{\multicolumn{2}{|c|}{$\frac{\text { First year tertiary students }}{2009-2012(n=368)}$}} & \multirow{2}{*}{\multicolumn{2}{|c|}{$\frac{\text { First year bachelor students }}{2009-2012(n=242)}$}} \\
\hline & & & & \\
\hline & Mean estimate $(95 \% \mathrm{Cl})$ & $P$ value & Mean estimate (95 \% Cl) & $P$ value \\
\hline \multicolumn{5}{|c|}{ GPA Core 4 Courses } \\
\hline \multicolumn{5}{|c|}{ Any 2 sciences $(\mathrm{NCEA}, \mathrm{CIE}, \mathrm{IB})^{* *}$} \\
\hline No & & & 0.00 & \\
\hline Yes & & & $1.12(0.30,1.94)$ & $0.0082^{*}$ \\
\hline \multicolumn{5}{|c|}{ Followed advice } \\
\hline No & & & 0.00 & \\
\hline Yes & & & $1.10(0.36,1.84)$ & 0.0040 \\
\hline \multicolumn{5}{|c|}{ CertHSc MMI } \\
\hline \multicolumn{5}{|c|}{ Whānau Support } \\
\hline $\mathrm{FC}^{\mathrm{a}}$ & & & 0.00 & \\
\hline SC & & & $0.75(-0.15,1.61)$ & 0.0909 \\
\hline$M C$ & & & $-0.34(-3.48,2.79)$ & 0.8300 \\
\hline \multicolumn{5}{|c|}{ Academic Preparation } \\
\hline FC & & & 0.00 & \\
\hline SC & & & $-0.32(-1.30,0.67)$ & 0.5259 \\
\hline MC & & & $0.91(-0.86,2.67)$ & 0.3145 \\
\hline \multicolumn{5}{|c|}{ Career Aspirations } \\
\hline FC & & & 0.00 & \\
\hline SC & & & $-1.37(-2.26,-0.48)$ & $0.0029^{*}$ \\
\hline$M C$ & & & $1.21(-0.62,3.04)$ & 0.1961 \\
\hline \multicolumn{5}{|c|}{ Student Information } \\
\hline FC & & & 0.00 & \\
\hline SC & & & $-0.66(-1.57,0.24)$ & 0.1532 \\
\hline MC & & & $2.60(0.02,5.17)$ & 0.0490 \\
\hline \multicolumn{5}{|c|}{ Bachelor MMI } \\
\hline \multicolumn{5}{|c|}{ Whānau Support } \\
\hline FC & & & 0.00 & \\
\hline SC & & & $0.09(-0.67,0.85)$ & 0.8159 \\
\hline $\mathrm{MC}$ & & & $0.52(-.076,1.81)$ & 0.4249 \\
\hline \multicolumn{5}{|c|}{ Academic Preparation } \\
\hline FC & & & 0.00 & \\
\hline SC & & & $-0.10(-0.86,0.65)$ & 0.7887 \\
\hline$M C$ & & & $-0.10(-1.12,0.92)$ & 0.8484 \\
\hline \multicolumn{5}{|c|}{ Career Aspirations } \\
\hline FC & & & 0.00 & \\
\hline SC & & & $-0.77(-1.45,-0.10)$ & 0.0256 \\
\hline$M C$ & & & $-0.74(-1.66,0.18)$ & 0.1179 \\
\hline \multicolumn{5}{|c|}{ Student Information } \\
\hline FC & & & 0.00 & \\
\hline SC & & & $-0.22(-0.89,0.46)$ & 0.5299 \\
\hline$M C$ & & & $-1.19(-2.29,-0.10)$ & 0.0331 \\
\hline
\end{tabular}


Table 5 Univariate regression analysis results - GPA core 4 courses (Continued)

\begin{tabular}{|c|c|c|}
\hline \multicolumn{3}{|l|}{ Continuous variables } \\
\hline \multicolumn{3}{|l|}{ School Results (NCEA)* } \\
\hline Rank Score & $0.34(0.24,0.46)$ & $<0.0001^{*}$ \\
\hline L3 English^ & $-0.03(-0.13,0.06)$ & 0.5145 \\
\hline L3 Biology & $0.04(-0.06,0.14)$ & 0.4349 \\
\hline L3 Chemistry & $-0.05(-0.14,0.04)$ & 0.2837 \\
\hline L3 Physics & $0.07(0.001,0.14)$ & 0.0528 \\
\hline L3 Maths & $0.04(-0.003,0.09)$ & 0.0734 \\
\hline MAPAS Maths test (per $10 \%$ increase) & $0.26(0.09,0.44)$ & $0.0039^{*}$ \\
\hline MAPAS English test(per $10 \%$ increase) & $0.03(-0.24,0.29)$ & 0.8523 \\
\hline
\end{tabular}

$\wedge$ L3 subject missing data includes those NCEA applicants who did not enrol in that particular subject

${ }^{\text {a }}$ FC Few concerns

*Adjusted for MAPAS interview year, gender, ancestry and school decile. For GPA (a continuous outcome variable), its mean change associated with the change in alinear predictor was estimated with $95 \%$ confidence interval. For a continuous predictor variable, this gave the difference in means with either 20 point (NCEA Rank Score) or $10 \%$ (MAPAS Maths percentage mark) increase in the predictor. For a categorical predictor, this gave the difference in means between the current and reference categories (i.e. yes vs. no). The null hypothesis was that there was no change in the mean response (i.e. $\Delta=0$ )

${ }^{* *} \mathrm{NCEA}=$ National Certificate in Educational Achievement, $\mathrm{CIE}=$ Cambridge International Exam, IB $=$ International Baccalaureate

\section{MMI}

Over $80 \%$ of all students from both cohorts were assessed as having few concerns for CertHSc-level entry across the four MMI stations. Forty-four percent of all First Year Tertiary students were assessed as having some or major concerns for bachelor-level entry at the Academic Preparation and Student Information MMI stations. For First Year Bachelor students, the stations with the highest proportion of some or major concerns for bachelor-level entry were Career Aspirations (48 \%) and Student Information (39\%) (Table 3).

\section{School results}

The average NCEA rank score (out of a total of 320) was 190.5 (SD 51.3) for First Year Tertiary and 201.8 (SD 52.7) for First Year Bachelor students. Both averages fall below requirements for guaranteed entry within FMHS (set at a rank score between 210 - 250 depending on the programme). The average number of subject credits for both cohorts were 0.3-3.4 credits below requirements for guaranteed entry (i.e. 16 - 18 subject credits depending on programme) (Table 3). At least two thirds of all students admitted into either the CertHSc or bachelor programmes had taken two or more science subjects in their final year of secondary school (Table 3).

\section{MAPAS recommendations}

For First Year Tertiary students, MAPAS recommended CertHSc to $72 \%$ of all students, followed by Bachelor (26\%) and Not FMHS (2 \%). For First Year Bachelor students, $58 \%$ were recommended to start at the CertHSc level, followed by $39 \%$ Bachelor and $3 \%$ Not FMHS (Table 3).

\section{Followed MAPAS advice}

Over $83 \%$ of all students followed MAPAS advice regarding the best starting point for success with only 12 $17 \%$ of students from each cohort not following their final MAPAS recommendation (Table 3).

\section{Outcome variables}

GPA All eight courses and core 4 courses

The average GPA for all eight courses (out of a total of 9) was 4.3 (SD 2.0) for First Year Tertiary and 4.1 (SD 2.1) for First Year Bachelor students. The average GPA achieved for the Core 4 Courses was 3.8 (SD 2.4) for First Year Bachelor students.

\section{Passes All eight courses and passes All core 4 courses}

Seventy-five percent of First Year Tertiary students and $60 \%$ of First Year Bachelor students passed all eight courses. Sixty-four percent of First Year Bachelor students passed all Core 4 Courses (Table 2).

\section{Multiple regression analysis}

First year tertiary - GPA

As shown in Table 8, all predictors had a statistically significant effect on First Year Tertiary GPA, with the most significant predictor being NCEA Rank Score, then MAPAS Advice Followed, Any 2 Sciences and MAPAS Mathematics Test results. First year Tertiary GPA increased by an average of 0.3 (out of a total 9) for every 20 point increase in NCEA Rank Score (CI: 0.18-0.34; $p<0.0001)$. Students who followed MAPAS advice had on average a GPA that was 1.2 points higher (out of a total 9) than students who did not (CI: 0.57-1.78; $p=0.0002)$. 
Table 6 Univariate regression analysis results - passes all eight courses

\begin{tabular}{|c|c|c|c|c|}
\hline \multirow[t]{3}{*}{ Predictors } & \multicolumn{2}{|c|}{ First year tertiary students } & \multicolumn{2}{|c|}{ First year bachelor students } \\
\hline & \multicolumn{2}{|l|}{$2009-2012(n=368)$} & \multicolumn{2}{|l|}{$2009-2012(n=242)$} \\
\hline & Odds ratio $(95 \% \mathrm{Cl})$ & Overall $P$ value & Odds ratio $(95 \% \mathrm{Cl})$ & Overall $P$ value \\
\hline \multicolumn{5}{|c|}{ Passes All Eight Courses } \\
\hline \multicolumn{5}{|c|}{ Any 2 sciences $(\mathrm{NCEA}, \mathrm{CIE}, \mathrm{IB})^{* *}$} \\
\hline No & 1.00 & & 1.00 & \\
\hline Yes & $2.52(1.32,4.83)$ & $0.005^{*}$ & $1.90(0.87,4.15)$ & 0.106 \\
\hline \multicolumn{5}{|c|}{ Followed advice } \\
\hline No & 1.00 & & 1.00 & \\
\hline Yes & $3.30(1.67,6.52)$ & $0.001^{*}$ & $1.97(0.98,3.98)$ & 0.058 \\
\hline \multicolumn{5}{|c|}{ CertHSc MMI } \\
\hline \multicolumn{5}{|c|}{ Whānau Support } \\
\hline$F C^{b}$ & 1.00 & & 1.00 & \\
\hline SC & $1.21(0.59,2.49)$ & & $1.60(0.68,3.72)$ & \\
\hline MC & $0.19(0.03,1.07)$ & 0.130 & $0.64(0.04,11.26)$ & 0.520 \\
\hline \multicolumn{5}{|c|}{ Academic Preparation } \\
\hline FC & 1.00 & & 1.00 & \\
\hline SC & $0.81(0.39,1.68)$ & & $0.89(0.35,2.27)$ & \\
\hline MC & $1.67(0.39,7.18)$ & 0.642 & $1.52(0.28,8.29)$ & 0.850 \\
\hline \multicolumn{5}{|c|}{ Career Aspirations } \\
\hline FC & 1.00 & & 1.00 & \\
\hline SC & $0.47(0.24,0.91)$ & & $0.38(0.14,0.80)$ & \\
\hline$M C$ & $0.47(0.10,2.13)$ & 0.061 & $1.32(0.22,7.87)$ & $0.042^{*}$ \\
\hline \multicolumn{5}{|c|}{ Student Information } \\
\hline FC & 1.00 & & 1.00 & \\
\hline SC & $1.26(0.61,2.59)$ & & $1.15(0.47,2.83)$ & \\
\hline$M C$ & $4.11(0.46,36.87)$ & 0.395 & $>999.999^{a}$ & 0.951 \\
\hline \multicolumn{5}{|c|}{ Bachelor MMI } \\
\hline \multicolumn{5}{|c|}{ Whānau Support } \\
\hline FC & 1.00 & & 1.00 & \\
\hline SC & $0.78(0.43,1.41)$ & & $0.79(0.39,1.63)$ & \\
\hline$M C$ & $0.79(0.29,2.14)$ & 0.686 & $1.65(0.46,5.95)$ & 0.541 \\
\hline \multicolumn{5}{|c|}{ Academic Preparation } \\
\hline FC & 1.00 & & 1.00 & \\
\hline SC & $1.58(0.82,3.05)$ & & $0.86(0.42,1.78)$ & \\
\hline$M C$ & $1.02(0.48,2.16)$ & 0.326 & $0.90(0.35,2.33)$ & 0.920 \\
\hline \multicolumn{5}{|c|}{ Career Aspirations } \\
\hline FC & 1.00 & & 1.00 & \\
\hline SC & $0.88(0.49,1.58)$ & & $0.57(0.30,1.08)$ & \\
\hline$M C$ & $0.77(0.36,1.64)$ & 0.791 & $0.74(0.31,1.77)$ & 0.228 \\
\hline \multicolumn{5}{|c|}{ Student Information } \\
\hline FC & 1.00 & & 1.00 & \\
\hline SC & $0.82(0.46,1.47)$ & & $0.77(0.41,1.47)$ & \\
\hline$M C$ & $0.97(0.41,2.31)$ & 0.799 & $0.50(0.18,1.38)$ & 0.375 \\
\hline
\end{tabular}


Table 6 Univariate regression analysis results - passes all eight courses (Continued)

\begin{tabular}{|c|c|c|c|c|}
\hline \multicolumn{5}{|l|}{ Continuous variables } \\
\hline \multicolumn{5}{|l|}{ School Results (NCEA)* } \\
\hline Rank Score (per 20 pt increase) & $1.08(0.96,1.20)$ & 0.178 & $1.35(1.17,1.54)$ & $<0.0001^{*}$ \\
\hline L3 English^ & $1.003(0.87,1.16)$ & 0.971 & $0.95(0.81,1.10)$ & 0.485 \\
\hline L3 Biology & $1.04(0.90,1.20)$ & 0.575 & $1.19(0.99,1.43)$ & 0.060 \\
\hline L3 Chemistry & $0.96(0.84,1.11)$ & 0.602 & $0.78(0.65,0.94)$ & $0.010^{*}$ \\
\hline L3 Physics & $1.15(1.01,1.31)$ & $0.039^{*}$ & $1.10(0.95,1.28)$ & 0.196 \\
\hline L3 Maths & $1.06(0.98,1.15)$ & 0.167 & $1.23(1.06,1.44)$ & $0.008^{*}$ \\
\hline MAPAS Maths test (per $10 \%$ increase) & $1.17(1.01,1.36)$ & $0.033^{*}$ & $1.19(1.02,1.42)$ & $0.032^{*}$ \\
\hline MAPAS English test(per $10 \%$ increase) & $0.94(0.75,1.17)$ & 0.595 & $0.84(0.65,1.09)$ & 0.202 \\
\hline
\end{tabular}

\section{First year tertiary - passes All courses}

The odds of passing all eight courses was 5.4 times higher for those students who followed MAPAS advice versus those students who did not (CI: 2.36-12.39; $p<0.0001$ ) (Table 8). The odds of passing all eight courses was 2.3 times higher for those students who had exposure to Any 2 Sciences versus those students who did not (CI: $1.15-4.61 ; p=0.019$ ) (Table 8).

\section{First year bachelor - GPA}

For every 20 point increase in NCEA Rank Score, the GPA achieved by First Year Bachelor students increased by an average of 0.4 for all 8 courses (CI: $0.30-0.50 ; p<0.0001$ ) and for Core 4 courses (CI: 0.26-0.50; $p<0.0001$ ) (Table 7). Students who followed MAPAS advice had on average a GPA that was 1.1 points higher than students who did not follow MAPAS advice for all eight courses (CI: 0.45-1.73; $p=0.0009$ ) and Core 4 courses (CI: 0.60-2.04; $p=0.0004$ ) (Table 8).

\section{First year bachelor - passes All courses}

A 20 point increase in NCEA Rank Score increased the odds of passing all first year bachelor courses by a factor of 1.5 (CI: $1.24-1.74 ; p<0.0001$ ), with similar results for passing all Core 4 courses (Table 8 ). The odds of passing all first year bachelor courses (CI: 1.45-7.69; $p=0.005$ ) and all Core 4 courses (CI: $1.39-7.69 ; p=0.007$ ) was 3.3 times higher for those students who followed MAPAS advice versus those students who did not (Table 9).

\section{Discussion}

Our findings confirm that the MAPAS admissions process is strongly associated with positive academic outcomes in the first year of tertiary study. Our results reinforce the evidencebase showing a strong association between secondary school performance via NCEA rank score (a marker of the quality of grades achieved) and positive tertiary academic outcomes [35]. The existing literature base has also been extended, given our identification of a strong association between exposure to two or more senior science subjects (a marker of breadth of knowledge) and first year academic outcomes. Similar to other studies, our findings show that the number of credits achieved within NCEA subjects appear to be less strongly correlated with tertiary outcomes [35].

Overall, our findings suggest that there is value in providing a comprehensive admissions process for indigenous and ethnic minority students applying under equity targeted admission programmes. Students admitted into tertiary institutions under targeted admission programmes have been shown to experience peer/educator stigma and 'everyday racism'. Demonstrating the effectiveness of targeted admission programmes may assist some indigenous and ethnic minority students to override this societal (and potentially internalised) stigma to receive the benefits that targeted admission programmes have to offer.

Increasing the odds of passing all first year courses has relevance for all students. This is important for applicants pursuing medicine as even small increments in first year bachelor GPA, particularly within the Core 4 courses used for medical selection, may have a profound impact on potential selection $[12,19]$. A student's progress towards completion of total point requirements within their degree has been shown to improve student retention and increase the likelihood of degree completion [36]. Aligning MAPAS admission to a comprehensive process focussed on achieving equity in access and performance is likely to have 
Table 7 Univariate regression analysis results: passes all core 4 courses

\begin{tabular}{|c|c|c|c|c|}
\hline \multirow[t]{3}{*}{ Predictors } & \multirow{2}{*}{\multicolumn{2}{|c|}{$\frac{\text { First year tertiary students }}{2009-2012(n=368)}$}} & \multicolumn{2}{|c|}{ First year bachelor students } \\
\hline & & & \multicolumn{2}{|l|}{$2009-2012(n=242)$} \\
\hline & Odds ratio $(95 \%$ Cl) & Overall $P$ value & Odds ratio $(95 \% \mathrm{Cl})$ & Overall $P$ value \\
\hline \multicolumn{5}{|c|}{ Passes All Core 4 Courses } \\
\hline \multicolumn{5}{|c|}{ Any 2 sciences $(\mathrm{NCEA}, \mathrm{CIE}, \mathrm{IB})^{* *}$} \\
\hline No & & & 1.00 & \\
\hline Yes & & & $2.57(1.16,5.68)$ & $0.020^{*}$ \\
\hline \multicolumn{5}{|c|}{ Followed advice } \\
\hline No & & & 1.00 & \\
\hline Yes & & & $1.83(0.90,3.71)$ & 0.095 \\
\hline \multicolumn{5}{|c|}{ CertHSC MMI } \\
\hline \multicolumn{5}{|c|}{ Whānau Support } \\
\hline $\mathrm{FC}^{\mathrm{a}}$ & & & 1.00 & \\
\hline SC & & & $1.51(0.64,3.57)$ & \\
\hline MC & & & $0.54(0.03,9.69)$ & 0.581 \\
\hline \multicolumn{5}{|c|}{ Academic Preparation } \\
\hline FC & & & 1.00 & \\
\hline SC & & & $0.79(0.31,2.03)$ & \\
\hline MC & & & $1.35(0.25,7.38)$ & 0.818 \\
\hline \multicolumn{5}{|c|}{ Career Aspirations } \\
\hline FC & & & 1.00 & \\
\hline SC & & & $0.36(0.15,0.84)$ & \\
\hline$M C$ & & & $1.21(0.19,7.52)$ & 0.059 \\
\hline \multicolumn{5}{|c|}{ Student Information } \\
\hline FC & & & 1.00 & \\
\hline SC & & & $1.03(0.42,2.54)$ & \\
\hline$M C$ & & & $>999.999^{\dagger}$ & 0.998 \\
\hline \multicolumn{5}{|c|}{ Bachelor MMI } \\
\hline \multicolumn{5}{|c|}{ Whānau Support } \\
\hline FC & & & 1.00 & \\
\hline SC & & & $0.70(0.34,1.46)$ & \\
\hline MC & & & $1.51(0.41,5.53)$ & 0.453 \\
\hline \multicolumn{5}{|c|}{ Academic Preparation } \\
\hline FC & & & 1.00 & \\
\hline SC & & & $0.76(0.36,1.59)$ & \\
\hline$M C$ & & & $1.01(0.38,2.66)$ & 0.737 \\
\hline \multicolumn{5}{|c|}{ Career Aspirations } \\
\hline $\mathrm{FC}$ & & & 1.00 & \\
\hline SC & & & $0.54(0.28,1.05)$ & \\
\hline MC & & & $0.60(0.25,1.46)$ & 0.175 \\
\hline \multicolumn{5}{|c|}{ Student Information } \\
\hline FC & & & 1.00 & \\
\hline SC & & & $0.69(0.36,1.32)$ & \\
\hline MC & & & $0.44(0.16,1.24)$ & 0.240 \\
\hline
\end{tabular}


Table 7 Univariate regression analysis results: passes all core 4 courses (Continued)

\begin{tabular}{|c|c|c|}
\hline \multicolumn{3}{|l|}{ Continuous variables } \\
\hline \multicolumn{3}{|l|}{ School Results (NCEA)* } \\
\hline Rank Score & $1.37(1.20,1.57)$ & $<0.0001^{*}$ \\
\hline L3 English^ & $1.01(0.83,1.23)$ & 0.921 \\
\hline L3 Biology & $1.20(0.95,1.51)$ & 0.134 \\
\hline L3 Chemistry & $0.85(0.70,1.03)$ & 0.089 \\
\hline L3 Physics & $1.10(0.94,1.29)$ & 0.213 \\
\hline L3 Maths & $1.27(1.04,1.54)$ & $0.017^{*}$ \\
\hline MAPAS Maths test (per $10 \%$ increase) & $1.21(1.02,1.42)$ & $0.029^{*}$ \\
\hline MAPAS English test(per $10 \%$ increase) & $0.99(0.96,1.01)$ & 0.283 \\
\hline
\end{tabular}

*Adjusted for MAPAS interview year, gender, ancestry and school decile. For Passes All Courses (a binary outcome variable), the odds ratio (OR) associated with the change in a linear predictor was estimated with $95 \%$ confidence interval. For a continuous predictor, this indicated the difference in ratio of two odds with either 20 point (NCEA Rank Score) or $10 \%$ (MAPAS Maths test) increase in the predictor, relative to the odds with no increase. For a categorical predictor, this indicated the difference in odds between the current and reference categories (e.g. the odds of Passes All Courses with exposure to Any 2 Sciences, relative to the odds of not having exposure to Any 2 Sciences). The null hypothesis was that there was no change in the odds (i.e. OR $=1$ )

**NCEA = National Certificate in Educational Achievement, $\mathrm{CIE}=$ Cambridge International Exam, IB = International Baccalaureate

${ }^{a}$ FC Few concerns, SMC Some or major concerns

${ }^{\dagger}$ Insufficient data available for analysis

contributed to the recent increase in numbers and improved performance observed for Māori and Pacific students within the FMHS [5, 37].

Our data suggests secondary schooling is yet to demonstrate the ability to prepare Māori and Pacific students adequately for tertiary health professional study. Both teaching and subject selection are critical factors. Māori and Pacific students and their families are not to blame for the observed inequities in secondary education. Rather, Māori and Pacific students and their families

Table 8 Multiple regression analysis results - linear regression ${ }^{a}$

\begin{tabular}{|c|c|c|c|c|}
\hline \multirow[t]{3}{*}{ Multivariate analysis results } & \multicolumn{2}{|l|}{ First year tertiary students } & \multicolumn{2}{|l|}{ First year bachelor students } \\
\hline & \multicolumn{2}{|l|}{$2009-2012(n=368)$} & \multicolumn{2}{|l|}{$2009-2012(n=242)$} \\
\hline & Mean estimate $(95 \% \mathrm{Cl})$ & $P$ value & Mean estimate $(95 \% \mathrm{Cl})$ & $P$ value \\
\hline \multicolumn{5}{|l|}{ GPA Eight Courses } \\
\hline NCEA Rank Score (per 20 point increase) & $0.26(0.18,0.34)$ & $<0.0001$ & $0.40(0.30,0.50)$ & $<0.0001$ \\
\hline \multicolumn{5}{|l|}{ Followed MAPAS advice } \\
\hline No & 0.00 & & 0.00 & \\
\hline Yes & $1.17(0.57,1.78)$ & 0.0002 & $1.09(0.45,1.73)$ & 0.0009 \\
\hline \multicolumn{5}{|l|}{ Any 2 sciences } \\
\hline No & 0.00 & & 0.00 & \\
\hline Yes & $0.65(0.15,1.15)$ & 0.0116 & $0.39(-0.29,1.08)$ & 0.2603 \\
\hline MAPAS Maths test (per $10 \%$ increase) & $0.14(0.02,0.26)$ & 0.0186 & $0.08(-0.07,0.22)$ & 0.2885 \\
\hline \multicolumn{5}{|l|}{ GPA Core 4 Courses } \\
\hline NCEA Rank Score (per 20 point increase) & - & - & $0.38(0.26,0.50)$ & $<0.0001$ \\
\hline Followed MAPAS advice & - & - & & \\
\hline No & & & 0.00 & \\
\hline Yes & - & - & $1.14(0.60,2.04)$ & 0.0004 \\
\hline Any 2 sciences & - & - & & \\
\hline No & & & 0.00 & \\
\hline Yes & - & - & $0.64(-0.13,1.41)$ & 0.1027 \\
\hline MAPAS Maths test (per $10 \%$ increase) & - & - & $0.15(-0.02,0.31)$ & 0.0765 \\
\hline
\end{tabular}


Table 9 Multiple regression analysis results - logistic regression ${ }^{a}$

\begin{tabular}{|c|c|c|c|c|}
\hline \multirow[t]{3}{*}{ Multivariate analysis results } & \multicolumn{2}{|c|}{ First year tertiary students } & \multicolumn{2}{|c|}{ First year bachelor students } \\
\hline & \multicolumn{2}{|l|}{$2009-2012(n=368)$} & \multicolumn{2}{|l|}{$2009-2012(n=242)$} \\
\hline & Odds ratio $(95 \% \mathrm{Cl})$ & $P$ value & Odds ratio $(95 \% \mathrm{Cl})$ & $P$ value \\
\hline \multicolumn{5}{|l|}{ Passes All Eight Courses } \\
\hline NCEA Rank Score (per 20 point increase) & $1.10(0.98,1.27)$ & 0.112 & $1.46(1.24,1.74)$ & $<0.0001$ \\
\hline \multicolumn{5}{|l|}{ Followed MAPAS advice } \\
\hline No & 1.00 & & 1.00 & \\
\hline Yes & $5.40(2.36,12.39)$ & $<0.0001$ & $3.34(1.45,7.69)$ & 0.005 \\
\hline \multicolumn{5}{|l|}{ Any 2 sciences } \\
\hline No & 1.00 & & 1.00 & \\
\hline Yes & $2.30(1.15,4.61)$ & 0.019 & $1.36(0.55,3.33)$ & 0.504 \\
\hline MAPAS Maths test (per $10 \%$ increase) & $1.13(0.95,1.33)$ & 0.179 & $1.08(0.90,1.32)$ & 0.392 \\
\hline \multicolumn{5}{|l|}{ Passes All Core 4 Courses } \\
\hline NCEA Rank Score (per 20 point increase) & - & - & $1.48(1.24,1.74)$ & $<0.0001$ \\
\hline Followed MAPAS advice & - & - & & \\
\hline No & & & 1.00 & \\
\hline Yes & - & - & $3.27(1.39,7.69)$ & 0.0067 \\
\hline Any 2 sciences & - & - & & \\
\hline No & & & 1.00 & \\
\hline Yes & - & - & $1.95(0.78,4.84)$ & 0.1513 \\
\hline MAPAS Maths test (per $10 \%$ increase) & - & - & $1.10(0.91,1.34)$ & 0.3156 \\
\hline
\end{tabular}

${ }^{a}$ Adjusted for MAPAS interview year, gender, ancestry and school decile. For Passes All Courses (a binary outcome variable), the odds ratio (OR) associated with the change in a linear predictor was estimated with $95 \%$ confidence interval. For a continuous predictor, this indicated the difference in ratio of two odds with either 20 point (NCEA Rank Score) or $10 \%$ (MAPAS Maths test) increase in the predictor, relative to the odds with no increase. For a categorical predictor, this indicated the difference in odds between the current and reference categories (e.g. the odds of Passes All Courses with exposure to Any 2 Sciences, relative to the odds of not having exposure to Any 2 Sciences). The null hypothesis was that there was no change in the odds (i.e. OR=1)

should receive greater support to navigate NCEA subject selection and ensure that students achieve the right number and quality of credits [38]. This is consistent with international evidence showing that indigenous and ethnic minority students are less likely to receive highquality careers or university advice [38, 39] and in some instances may be actively discouraged from pursuing a health professional career [2].

Based on our findings, it appears that the secondary education sector is failing to ensure that indigenous and ethnic minority students are 'university-ready' for health-professional study. Unfortunately, this is not a new issue $[5,14,40,41]$ and nor is it unique to New Zealand [3, 42]. Action by secondary schools and educators to address their own role in the creation and maintenance of ethnic inequities in academic outcomes is recommended [43]. Likewise, tertiary institutions are expected to be part of the solution [44]. Pechenkina \& Anderson (2011) call for "more effective institutional response to the lack of adequate preparation of indigenous students... via greater investment in the pipeline and provision of transitioning programmes" (p. 5-6). Our findings further support the delivery of bridging/foundation programmes targeting indigenous and ethnic minority students.

\section{Strengths}

This study explores a unique application of the MMI within an equity-targeted context $[14,26]$. Although we identified varied associations between individual MMI stations and academic outcomes, we believe that our overall findings support maintaining the MMI within the MAPAS admissions process. This reflects the strong association observed between following MAPAS advice (a predictor variable that is determined by the combined assessment of all results) and higher academic outcomes.

Using both cognitive (e.g. NCEA school results, MAPAS Maths and English test) and non-cognitive (e.g. MMI results) tools for student selection within the total MAPAS admission process supports a widening participation agenda and is consistent with recommendations to use more inclusive selection tools [10, 45-47]. This is particularly important when assessing the potential of alternative admission or older applicants who may possess 
maturity shown to be positively associated with tertiary programme completion $[36,48]$.

\section{Limitations}

This study has a number of limitations. The analysis relied on secondary data and is therefore limited by the quality of data sources. However, combining central university and MAPAS datasets has reduced the potential for data misclassification by using verified ancestry and increased the admission variables available for analysis $[49,50]$. Our research was limited to first-year outcomes due to resource and time constraints. Ideally, the effect of predictor variables on long-term outcomes across all FMHS programmes should be examined. Comparing academic outcomes across all ethnic groups may also highlight issues of disadvantage and privilege [51]. This research is in progress and is drawing on the methods developed within this study. We acknowledge that combining Māori and Pacific data is not ideal from an indigenous rights or Pacific-centric perspective. However, this is consistent with our methodological approach as it maximises statistical power (to aid student success) and supports a structural critique of the effect of 'society' on 'ancestry' [14]. As the quantum of Mãori and Pacific data increases, further research should investigate Mãori-specific and Pacific-specific predictors of academic success.

\section{Conclusion}

Tertiary institutions committed to widening participation should prioritise the funding and delivery of a comprehensive, flexible and inclusive admissions process that includes alternative entry pathways for indigenous and ethnic minority applicants $[10,52,53]$.

\section{Ethical approval}

This project was approved by the University of Auckland Human Participants Ethics Committee, Ref 8110.

\section{Endnotes}

${ }^{1}$ Completion of the first year of study within either the Bachelor of Health Sciences or the Bachelor of Science (Biomedicine) programme is required for an undergraduate application to the medical programme at the UoA

${ }^{2}$ For additional information, see previous publications 5 . Curtis E, Reid P. Indigenous health workforce development: Challenges and successes of the Vision 20: 20 programme. Australian \& New Zealand Journal of Surgery. 2013;83(2013):49-54, 13. Curtis E, Reid P, Jones R. Decolonising the Academy: The process of representing indigenous health in tertiary teaching and learning. In: Cram F, Phillips H, Sauni P, Tuagalu C, editors. Mãori and Pasifika Higher Education Horizons. Bingley, U.K.: Emerald Group Publishing Limited; 2014. p. 147-66, 14. Curtis, E., Wikaire, E., Jiang, Y., McMillan, L., Loto, R., Airini, \& Reid, P. (2015). A tertiary approach to improving equity in health: Quantitative analysis of the Māori and Pacific admission scheme (MAPAS) process, 2008-2012. International Journal for Equity in Health, 14(7). 10.1186/s12939-015-0133-7. or https://www.fmhs.auckland.ac.nz/en/faculty/for/futureundergraduates/maori-and-pacific-admission-scheme.html

${ }^{3}$ The National Certificate of Educational Achievement (NCEA) is the major assessment method used in New Zealand secondary schools. The NCEA Rank Score reflects the best 80 credits at Level 3 or higher, over a maximum of five approved subjects. It reflects a system of Grade Point Average and is used by the UoA to assist with admission to limited entry programmes 23. Shulruf B, Hattie J, Tumen S. New Zealand's standard-based assessment for secondary schools (NCEA): implications for policy makers. Asia Pacific Journal of Education. 2010;30(2).

${ }^{4}$ Exposure to a minimum of two final year secondary school science subjects is recommended for success within the CertHSc (alongside English and mathematics rich subjects). This variable includes secondary school results from NCEA, International Baccalaureate (IB) and Cambridge International Examinations (CIE).

${ }^{5}$ The Core 4 courses include: CHEM110 (Chemistry of the living world), POPLHLTH 111 (Population Health), MEDSCI 142 (Biology for Biomedicine Science: Organ Systems) and BIOSCI 107 (Biology for Biomedicine Science: Cellular Processes and Development).

\section{Abbreviations \\ CertHSc: Certificate in Health Sciences (Hikitia Te Ora); CIE: Cambridge International Exam; FMHS: Faculty of Medical and Health Sciences; GPA: Grade Point Average; IB: International Baccalaureate; KMR: Kaupapa Māori Research; MAPAS: Māori and Pacific Admission Scheme; NCEA: National Certificate of Educational Achievement; UoA: University of Auckland.}

\section{Competing interest}

The authors declare that they have no competing interest.

\section{Authors' contributions}

EC led the study design, methodological approach, interpretation of the data analysis, and drafted the manuscript. EW contributed to study design and provided research assistance to obtain and clean data variables. She contributed to drafting and revising the manuscript and was responsible for producing the data tables. YJ provided senior statistical expertise for data analysis. She contributed to drafting and revising the manuscript. LM provided junior statistical expertise and contributed to drafting and revising the manuscript. RL contributed to the study design and provided Pacific research methodological expertise in the drafting and revising of the manuscript. A provided senior Pacific educational and research expertise and contributed to drafting and revising the manuscript. PR provided senior Māori educational, institutional and KMR expertise and contributed to drafting and revising the manuscript. All authors read and approved the final manuscript for submission. All authors agreed to be accountable for all aspects of the work

\section{Authors' information}

EC (Te Arawa, FNZCPHM, MPH (Distinc), MBChB) is a specialist in public health medicine who has experience in research and policy concerned with 
eliminating ethnic and indigenous inequalities in health. Elana is a Senior Lecturer and the Director Vision 20:20 at Te Kupenga Hauora Māori, The University of Auckland. She is a postgraduate Doctor of Medicine (MD) candidate (exploring indigenous and ethnic minority health workforce development) and has ongoing research interests in ethnic inequities in service utilisation and health outcomes.

EW (Ngāti Hine, PGDipPH (Distinc), BHSc) is a Māori Physiotherapist who has experience in research concerned with Māori and Indigenous health workforce development, cultural competence, and psycho-oncology in Māori and Indigenous populations. Erena is currently completing a Masters in Public Health whilst working as Researcher at Te Kupenga Hauora Mãori, University of Auckland. Ongoing research interests include Māori health workforce development and addressing ethnic inequalities in health. YJ (Chinese, PhD) is a Senior Research Fellow at the Department of Statistic and Senior Statistical Consultant at the Statistical Consulting Centre (SCC), Faculty of Science, University of Auckland, New Zealand. Ongoing research interests include: randomised controlled trial design and analysis, national surveys, longitudinal and case-control studies with response-selective sampling and missing data problems.

LM (Pākehā, MSc, MMath) is an Assistant Analyst at the Statistical Consulting Centre (SCC), Faculty of Science, The University of Auckland, New Zealand. She is a PhD candidate in the Department of Mathematics and Statistics. RL (Samoa, PGDipPsych-Community, MSocS-Hons) proudly hails from the villages of Fagamalo and Avao (Savai'i) where he was raised as a young child. Rob is a Professional Teaching Fellow within Hikitia Te Ora - Certificate in Health Sciences programme at Te Kupenga Hauora Mãori, FMHS, UoA. Rob is a Registered Community Psychologist and his aspirations are firmly rooted in the wellbeing and development of Māori and Pacific communities in regards to identity and health.

A (PhD, MEd (Distinc), MBA, BA, DipTchg, CertARM) specialises in higher education research, with a particular focus on Pasifika, indigenous and under-served students. Airini has Samoan ancestry, has a national and international record in Pasifika education research and recognised expertise in Pasifika methodologies. Airini is Dean, Faculty of Human, Social and Educational Development, Thompson Rivers University, BC, Canada. With a view to informing further education system reform in New Zealand and internationally, as a Fulbright Scholar based in Washington DC Airini investigated how to convert education policy into better results for under-served students.

PR (Te Rarawa, DipComH, BSc, MBChB, DipObst, FNZCPHM) is Tumuaki and Head of Department of Mãori Health at the Faculty of Medical and Health Sciences, University of Auckland, New Zealand. She is a specialist in public health medicine and her research interests include analysing disparities between indigenous and non-indigenous citizens as a means of monitoring government commitment to indigenous rights.

\section{Acknowledgements}

The authors would like to thank members of the Te Hā Advisory Group: Dr Teuila Percival; Dr Vili Nosa; Dr Malakai Ofanoa; Associate Professor Mark Barrow; Lynley Pritchard; James Clark and Carolyn (Shaoxun) Huang. Andrew Sporle and Joanna Stewart are acknowledged for providing input into the early stages of project design from a statistical perspective. Dr Elana Curtis was supported by Te Kete Hauora, Ministry of Health (New Zealand) to conduct this research via the provision of a Research Fellowship (Contract 414953/337535/00). We also thank Ngā Pae o Te Māramatanga for their support for Erena Wikaire to attend and present these research findings at the Leaders in Indigenous Medical Education (LIME) Connection V conference in Darwin, Australia 2013.

\section{Author details}

${ }^{1}$ Te Kupenga Hauora Mãori, Faculty of Medical and Health Sciences, University of Auckland, Private Bag 92015, Auckland, New Zealand. ${ }^{2}$ Department of Statistics, Faculty of Science, University of Auckland, Private Bag 92015, Auckland, New Zealand. ${ }^{3}$ Faculty of Human, Social and Educational Development, Thompson Rivers University, Thompson, BC, Canada.

Received: 15 December 2014 Accepted: 20 October 2015

Published online: 03 November 2015

\section{References}

1. Whitehead G, Shah M, Nair C. Equity and excellence are not mutually exclusive: A discussion of academic standards in an era of widening participation. Quality Assurance in Education. 2013;21(3):299-310.

2. Hollow W, Buckley A, Patterson DG, Olsen P, Medora R, Morin L, et al. Clearing the Path to Medical School for American Indians and Alaska Natives: New Strategies. Washington: School of Medicine, University of Washington and WWAMI Centre for Health Workforce Studies; 2006.

3. Sullivan L. Missing person: Minorities in the health professions. A report of the Sullivan Commission on diversity in the healthcare workforce. 2004.

4. Cooney R, Kosoko-Lasaki O, Slattery B, Wilson MR. Proximal versus distal influences on underrepresented minority students pursuing health professional careers. J Natl Med Assoc. 2006;98(9):1471-5.

5. Curtis E, Reid P. Indigenous health workforce development: Challenges and successes of the Vision 20: 20 programme. Aust N Z J Surg. 2013;83(2013):49-54.

6. Bediako MR, McDermott BA, Bleich ME, Colliver JA. Ventures in education: A pipeline to medical education for minority and economically disadvantaged students. Acad Med. 1996;71(2):190-2.

7. Farrington S, Page S, DiGregorio KD. The things that matter: Understanding the factors that affect the participation and retention of Indigenous students in the Cadigal Program at the Faculty of Health Sciences, University of Sydney. In: Joint Annual Conference of the Australian Association for Research in Education (AARE) and New Zealand Association for Research in Education (NZARE). Melbourne; 1999.

8. Ratima M, Brown R, Garrett N, Wikaire E, Ngawati R, Aspin C, et al. Rauringa Raupa: Recruitment and rentention of Maori in the health and disability workforce. In: Division of Public Health and Psychosocial Studies, Faculty of Health and Environmental Sciences. Auckland: Taupua Waiora: AUT University; 2008.

9. Anderson M, Lavallee B. The development of the First Nations, Inuit and Metis medical workforce. Med J Aust. 2007;186(10):539-40

10. Powis D, Hamilton J, McManus IC. Widening access by changing the criteria for selecting medical students. Teach Teach Educ. 2007;23:1235-45.

11. Zhou Y-X, Zhao Z-T, Wan C-S, Peng C-H, Yang J, Ou C-Q. Predictors of firstyear GPA on medical students: A longitudinal study of 1285 matriculates in China. BMC Med Educ. 2014;14:87.

12. Poole P, Moriarty H, Wearn T, Wilkinson T, Weller J. Medical Student Selection in New Zealand: Looking to the future. N Z Med J. 2009;122(1306):88-100.

13. Curtis $E$, Reid $P$, Jones R. Decolonising the Academy: The process of re-presenting indigenous health in tertiary teaching and learning. In: Cram F, Phillips H, Sauni P, Tuagalu C, editors. Māori and Pasifika Higher Education Horizons. 15th ed. Bingley, U.K: Emerald Group Publishing Limited; 2014. p. 147-66.

14. Curtis E, Wikaire E, Jiang $Y$, McMillan L, Loto R, Airini, et al. A tertiary approach to improving equity in health: Quantitative analysis of the Māori and Pacific admission scheme (MAPAS) process, 2008-2012. Int J Equity Health. 2015:14:7.

15. Salmi J, Bassett R. The equity imperative in tertiary education: Promoting fairness and efficiency. Int Rev Educ. 2014;60(3):1-18.

16. Tinto, V. Taking Retention Seriously: rethinking the first year of college. NACADA Journal. 1997;19(2):5-9.

17. Fazey D, Fazey J. The potential for autonomy in learning: Perceptions of competence, motivation and locus of control in first-year undergraduate students. Stud Higher Educ. 2001;26(3):345-61.

18. Krause K-L, Coates $H$. Students' engagement in first-year university. Assess Eval High Educ. 2008;33(5):493-505.

19. Shulruf B, Poole P, Wang G, Rudland J, Wilkinson T. How well do selection tools predict performance later in a medical programme? Adv Health Sci Educ. 2012;17:615-26.

20. Zepke N, Leach L. Improving student engagement: Ten proposals for action. Active Learn High Educ. 2010;11(3):167-77.

21. Shulruf B, Hattie J, Tumen S. The predictability of enrolment and first-year university results from secondary school performance: The New Zealand National Certificate of Educational Achievement. Studies High Educ. 2008;33(6):685-98.

22. Shulruf B, Hattie J, Tumen S. Individual and school factors affecting students' participation and success in higher education. High Educ 2008;56:613-32.

23. Flett R, Gavala J. Influencial factors moderating academic enjoyment/ motivation and psychological well-being for Māori university students at Massey University. N Z J Psychology. 2005;34(1):52-7. 
24. The University of Auckland. The University of Auckland Faculty of Medical and Health Sciences undergraduate prospectus. Auckland: The University of Auckland; 2015. p. 2014.

25. Pau A, Jeevaratnam K, Chen Y, Fall A, Khoo C, Nadarajah V. The Multiple Mini-Interview (MMI) for student selection into health professoins training - A systematic review. Med Teach. 2013;35:1027-41.

26. Eva K, Rosenfeld J, Reiter H, Norman G. An admmission OSCE: The multiple mini-interview. Med Educ. 2004;38:314-26.

27. Harris S, Owen C. Discerning quality: Using the multiple mini-interview in student selection for the Australian National University Medical School. Med Educ. 2007:41:234-41

28. Brownell K, Lockyer J, Collin T, Lemay J. Introduction of the multiple mini interview into the admission process at the University of Calgary: Acceptability and feasibility. Med Teach. 2007;29:394-6.

29. Smith L. Decolonizing Methodologies: Research and Indigenous Peoples. 2nd ed. London \& New York: Zed Books; 2012

30. Health Research Council of New Zealand. Guidelines for Pacific health research. In. Auckland: Health Research Council of New Zealand; 2005.

31. Eketone A. Theoretical underpinnings of Kaupapa Māori directed practice. MAI Review. 2008;1:1-11.

32. Reid P, Robson B. Understanding Health Inequities. In: Robson B, Harris R editors. Hauora: Māori Standards of Health IV A study of the years 20002005. Wellington: Te Rōpū Rangahau Hauora a Eru Pōmare; 2007. p. 3-10.

33. Valencia RR. The Evolution of Deficit Thinking: Educational Thought and Practice. Washington DC: The Palmer Press; 1997.

34. McKinley E, Madjar I. From Schools in Low-income Communities to University: Challenges of Transition For Māori and Pacific Students. In: Diversity in Higher Education Māori and Pasifika Higher Education Horizons. Volume 15, First edn. Edited by Cram F, Phillipa H, Sauni P, Tuagalu C. Bingley, UK: Emerald Group Publishing Limited; 2014: 241-252.

35. Shulruf $B$, Meisong L, McKimm J, Smith M. Breadth of knowledge vs. grade: What best predicts achievement in the first year of health sciences programmes? J Educ Eval Health Prof. 2012;9:7.

36. Tumen S, Shulruf B, Hattie J. Student pathways at the university: Patterns and predictors of completion. Studies High Educ. 2008;33(3):233-52.

37. Skene J, S E. Does access equal success? The critical role of the FYE in achieving equity in higher education. In: 12th Pacific Rim First Year in Higher Education Conference, 'Preparing for tomorrow today: The First Year as foundation'. Townsville: The University of Western Australia; 2009.

38. McKinley E, Madjar I. From schools in low-income communities to university. Māori and Pacifika higher education horizons. In: Māori and Pasifika higer education horizons (Diversity in higher education, Volume 15). edn. Edited by Cram F, Phillips H, Sauni P, Tuagalu C: Bingley: Emerald Group Publishing Limited; 2014: 1-19.

39. Drysdale M, Faulkner S, Chesters J. Footprints forwards: Better strategies for the recruitment, retention and support of Indigenous medical students. In. Monash University School of Rural Health, Moe; 2006.

40. Curtis E, Wikaire E, Stokes K, Reid P. Addressing indigenous health workforce inequities: A literature review exploring 'best' practice for recruitment into tertiary health programmes. Int J Equity Health. 2012;11:13.

41. Ratima M, Brown $\mathrm{R}$, Garrett N, Wikaire E, Ngawati R, Aspin C, Potaka U. Rauringa Raupa. Recruitment and retention of Māori in the health and disability workforce. In. Auckland: Taupua Waiora: Division of Public Health and Psychosocial Studies. Faculty of Health and Environmental Sciences: AUT University; 2008

42. Pechenkina E, Anderson I. Background paper on Indigenous Australian Higher Education: Trends, Initiatives and Policy Implications. Prepared for The Review of Higher Educaiton Access and Outcomes for Aboriginal and Torres Strait Islander People. Canberra: Commonwealth of Australia; 2011.

43. Bishop R, Berryman M, Tiakiwai S, Richardson C. Te Kotahitanga: The Experiences of Year 9 and 10 Māori Students in Mainstream Classrooms. Hamilton: University of Waikato; 2003.

44. Tertiary Education Commission. Initial Plan Guidance for 2013 Plans: Guidance for all TEOs. Wellington: Tertiary Education Commission; 2012.

45. Siu E, Reiter H. Overview: what's worked and what hasn't as a guide towards predictive admissions tool development. Adv in Health Sci Educ. 2009;14:759-75.

46. Wilkinson D, Zhang J, Byrne GHL, Ozolins 1Z, Parker M, Peterson R. Medical school selection criteria and the prediction of academic performance. Evidence leading to change in policy and practice at the University of Queensland. Med Educ. 2008;188:349-54.
47. Poole P, Shulruf B. Shaping the future medical workforce: take care with selection tools. J Prim Health Care. 2013:5(4):269-75.

48. O'Shea, S. Avoiding the manufacture of 'sameness': first-in family students, cultural capital and the higher education environment. Higher Education. 2015; ePub 16 September:1-20.

49. Ministry of Health. Ethnicity Data Protocols for the Health and Disability Sector. Wellington: Ministry of Health; 2004.

50. Tertiary Education Commission. Educational Performance Indicators: Definitions and Methodology. Measuring Student Achievement for Tertiary Education Organisations. All funds reported through SDR. Version 7. Wellington: Tertiary Education Commission; 2014.

51. McIntosh P. White privilege and Male privilege: A personal account of coming to see corresponsdences through work in women's studies. Working paper 189. In., vol. MA 02181. Wellesley: Wellesley College Center for Research on Women; 1988.

52. Salmi J, Bassett R. The equity imperative in tertiary education: Promoting fairness and efficiency. International Review of Education. 2014;60(3):1-18.

53. Clancy P, Goastellec G. Exploring Access and Equity in Higher Education: Policy and Performance in a Comparative Perspective. Higher Education Quarterly. 2007;61(2):136-54

\section{Submit your next manuscript to BioMed Central and take full advantage of:}

- Convenient online submission

- Thorough peer review

- No space constraints or color figure charges

- Immediate publication on acceptance

- Inclusion in PubMed, CAS, Scopus and Google Scholar

- Research which is freely available for redistribution 